\title{
ON THE ITERATIONS OF DIFFEOMORPHISMS WITHOUT $C^{0}-\Omega$-EXPLOSIONS: AN EXAMPLE
}

\author{
KEN SAWADA
}

\begin{abstract}
In this note we construct a diffeomorphism $f$ such that $f$ has no $C^{0}$ - $\Omega$-explosion but $f^{2}$ has $\Omega$-explosion.
\end{abstract}

The purpose of this note is to give an example of a diffeomorphism $f$ on a 2 -sphere $S^{2}$ such that $f$ has no $C^{0}-\Omega$-explosion but $f^{2}$ has $\Omega$-explosion. As noted below, this is accomplished by finding a diffeomorphism $f$ without $C^{0}$ - $\Omega$-explosion for which $\Omega(f) \neq \Omega\left(f^{2}\right)$.

Before proceeding to construct the example, we shall make a few observations on the iterations of diffeomorphisms without $C^{0}$ - $\Omega$-explosions:

A point $x \in S^{2}$ is said to be a chain recurrent point of $f$ if for any $\varepsilon>0$ there exists a sequence $\left\{x_{0}, \ldots, x_{n}\right\}$ of points on $S^{2}$ with $x_{0}=x_{n}=x$ and $d\left(f\left(x_{i}\right), x_{i+1}\right)$ $<\varepsilon$ where $d$ is a metric on $S^{2}$ (cf. [1]). We denote the sets of chain recurrent points and nonwandering points of $f$ by $R(f)$ and $\Omega(f)$ respectively. By definitions, it follows that $\Omega\left(f^{m}\right) \subset \Omega(f) \subset \Re(f)=\Re\left(f^{m}\right), m \neq 0$. In [2, Theorem 3.11] M. Shub showed that $f$ has no $C^{0}-\Omega$-explosion if and only if $\mathcal{R}(f)=\Omega(f)$. From the above facts, we have

Proposition. $A$ diffeomorphism $f^{m}$ has no $C^{0}$ - $\Omega$-explosion if and only if $f$ has no $C^{0}-\Omega$-explosion and $\Omega(f)=\Omega\left(f^{m}\right)$.

Hence for our purpose, it is sufficient to construct a diffeomorphism $f$ on $S^{2}$ such that $f$ has no $C^{0}-\Omega$-explosion and $\Omega(f) \neq \Omega\left(f^{2}\right)$, i.e., $R(f)=\Omega(f) \neq \Omega\left(f^{2}\right)$.

The author wishes to thank Professor H. Noguchi, Y. Togawa and K. Yano for helpful comments to this note.

The construction. At first we take a diffeomorphism $f^{\prime}$ on $S^{2}$ to be the time one map of the flow $\psi_{t}$ on $S^{2}$ as pictured in Figures 1 and 2 . Here Figures 1 and 2 show the flow $\psi_{t}$ on the upper and the lower hemispheres, $\mathrm{H}^{+}$and $\mathrm{H}^{-}$respectively, and $S^{1}$ is the equator, 0 and $0^{\prime}$ are the north and the south poles respectively, and $A^{\prime}$ is the antipodal point of $A$.

For $f^{\prime}$, there are two fixed points $A$ and $A^{\prime}$, two fixed sources 0 and $0^{\prime}$, and clearly $S^{1}$ is an invariant set. Furthermore let $f^{\prime}$ satisfy that $\left|\theta\left(f^{\prime}(x)\right)\right|<|\theta(x)|$ for any $x \in S^{2}-\left(S^{1} \cup 0 \cup 0^{\prime}\right)$ where $\theta(x)$ is the latitude of $x(-\pi / 2<\theta(x)<\pi / 2)$ and $|(\ldots)|$ is the absolute value of $(\ldots)$.

Received by the editors February 12, 1979 and, in revised form, May 16, 1979. AMS (MOS) subject classifications (1970). Primary 58F99; Secondary 57D50.

Key words and phrases. Diffeomorphisms, no $C^{0}-\Omega$-explosion. 


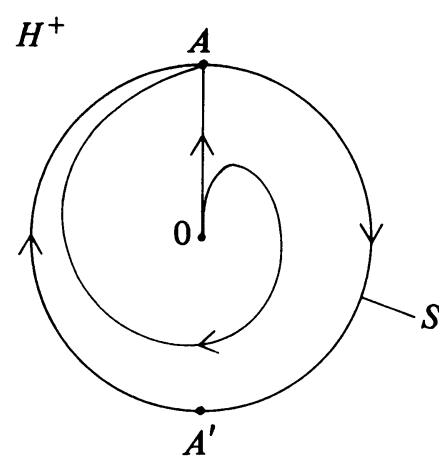

FIGURE 1

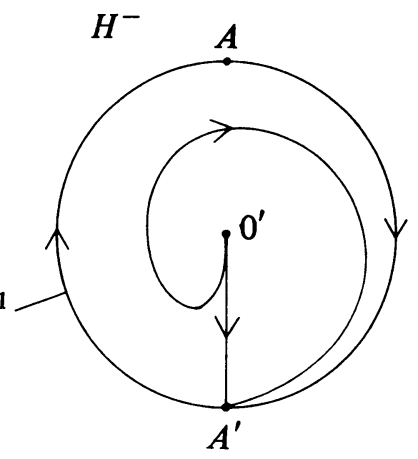

FIGURE 2

Now we construct a diffeomorphism $f$ on $S^{2}$ as follows; $f=\rho \circ f^{\prime}$ where $\rho$ is the map such that $\rho(x)$ is the antipodal point of $x$ for any $x \in S^{2}$. Note that $A, A^{\prime}$ are periodic points, $0,0^{\prime}$ are periodic sources of period 2 of $f$, and that $f$ satisfies

$$
|\theta(f(x))|<|\theta(x)| \text { for any } x \in S^{2}-\left(S^{1} \cup 0 \cup 0^{\prime}\right) .
$$

LEMMA. $\mathscr{R}(f)=\Omega(f) \neq \Omega\left(f^{2}\right)$.

Proof. We first show that $R(f)=0 \cup 0^{\prime} \cup S^{1}$. Clearly $0 \cup 0^{\prime} \cup S^{1} \subset \Re(f)$. Hence it suffices to show that $x \notin \Re(f)$ for $x \in S^{2}-\left(0 \cup 0^{\prime} \cup S^{1}\right)$. Let $\theta=$ $|\theta(x)|$ and $B=\left\{y \in S^{2}:|\theta(y)| \leqslant \theta\right\}$. Then by $\left(^{*}\right),|\theta(f(y))|<\theta$ for any $y \in B$. Since $B$ is compact, there exists $\varepsilon>0$ such that $\left|\theta\left(y^{\prime}\right)\right|<\theta$ for any $y^{\prime} \in U_{\varepsilon}(f(B))$ $\left(U_{e}(\ldots)\right.$ is an $\varepsilon$-neighborhood of $\left.(\ldots)\right)$. Let $\left\{x_{0}, \ldots, x_{m}\right\}$ be a sequence of points on $S^{2}$ with $x_{0}=x, d\left(f\left(x_{i}\right), x_{i+1}\right)<\varepsilon$. Then $x_{1} \in U_{\varepsilon}(f(B)) \subset B$ since $x_{0}=x$ $\in B$. By induction, $x_{m} \in U_{\varepsilon}(f(B))$ so that $\left|\theta\left(x_{m}\right)\right|<\theta=|\theta(x)|$. Therefore there exists no sequence $\left\{x_{0}, \ldots, x_{n}\right\}$ of points on $S^{2}$ with $x_{0}=x_{n}=x$ and $d\left(f\left(x_{i}\right), x_{i+1}\right)<\varepsilon$. Hence $x \notin \Re(f)$ and $R(f)=0 \cup 0^{\prime} \cup S^{1}$.

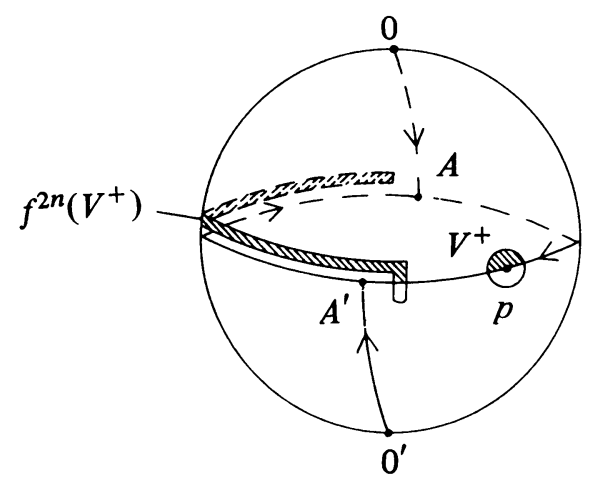

FIgURE 3

We next show that $R(f)=\Omega(f) \neq \Omega\left(f^{2}\right)$. Clearly $0,0^{\prime}, A, A^{\prime} \in \Omega(f)\left(\in \Omega\left(f^{2}\right)\right)$. Let $p \in S^{1}-\left(A \cup A^{\prime}\right), V$ be a neighborhood of $p$ and $V^{+}=V \cap\left(H^{+}-S^{1}\right)$. Without loss of generality, we may assume that $f^{2 n}(p) \rightarrow A^{\prime}$ as $n \rightarrow \infty$. Then 
$f^{2 n}\left(V^{+}\right)$is pressed toward $S^{1}$ as in Figure 3. Therefore for a sufficiently large $n$, $f^{2 n+1}(V) \cap V=f^{2 n+1}\left(V^{+}\right) \cap V \neq \varnothing$. Hence $p \in \Omega(f)$ and $\Re(f)=\Omega(f)$.

On the other hand, $\left(f^{2}\right)^{n}(V) \cap V=f^{2 n}(V) \cap V=\varnothing$. Hence $p \notin \Omega\left(f^{2}\right)$. Therefore $R(f)=\Omega(f) \neq \Omega\left(f^{2}\right)$.

Hence $f$ has no $C^{0}-\Omega$-explosion but $f^{2}$ has $\Omega$-explosion.

\section{REFERENCES}

1. C. Conley, The gradient structure of flow. I, IBM research, RC 3932 (\# 17806), Yorktown Heights, New York, July 17, 1972.

2. M. Shub, Stabilité globale des systemes dynamiques, Asterisque 56 (1976).

Department of Mathematics, Waseda University, Shmnjuku, Tokyo 160, JaPan 